\title{
SUPERPOSITION OF SEA ICE CLASSIFICATION BASED ON SYNTHETIC APERTURE RADAR IMAGES CONSIDERING UNDERLYING DRIFT
}

\author{
Maurice Wiercioch, Anja Frost, Suman Singha \\ Deutsches Zentrum für Luft- und Raumfahrt (DLR), Maritime Safety and Security Lab Bremen
}

\begin{abstract}
Using sea ice information generated from Synthetic Aperture Radar (SAR) products can increase the safety and efficiency of ship operations in ice-infested waters. For the purpose of operational sea ice classification, the results need to be highly reliable. The combination of multiple SAR acquisitions can be used to estimate the reliability of sea ice classification and to overcome existing limitations. In this paper, we present a new approach for comparing sea ice classification results from pairs of independent TerraSAR-X acquisitions and additionally overlay it with information on sea ice movement. For this purpose, we combine our processors for estimating sea ice drift and classification. The sea ice drift field is used to compensate the ice movement between two SAR acquisitions and differentiate areas of homogeneous and inhomogeneous ice zones. The processing chain is developed for operational usage in near real-time.
\end{abstract}

Index Terms - Synthetic Aperture Radar, sea ice, navigation, near real-time, drift, classification

\section{INTRODUCTION}

Ship navigation in polar regions is a challenge for humans and technology. Being remote of any opportunities for support, in an environment of fast changing weather and sea ice conditions, can create dangerous situations during maritime operations. Wind and ocean currents interact with the sea ice and can push floes together creating pressure ridges, which can be an insuperable obstacle for ships. Divergent forces can open up the sea ice cover, providing a faster and safer way through the ice. For this reason, knowledge about sea ice movement, coverage and ice-type distribution are indispensable for navigation in ice covered waters. SAR satellite products can provide this information.

Independent from cloud cover and darkness, SAR offers the opportunity to track sea ice and to distinguish between different ice types. In our previous work, we developed processors for the derivation of high resolution sea ice drift fields[1] and classification of different sea ice types[2]. Due to weather changes and deformation, the sea ice surface can change, which in turn has influence on the scattering properties of the sea ice. Also the incidence angle of the SAR acquisition has a large impact on the appearance of sea ice in the image and therefore on the quality of our processors. For the purpose of operationally supporting ship navigation with SAR based sea ice classification, the results need to be highly reliable. Combining co-located SAR products from different acquisition times can be used to estimate the reliability of sea ice classification and to overcome existing limitations. Due to the continuously sea ice movement, the superposition of sea ice information is only possible respecting the underlying drift. In general, this issue is solved due to pairs of SAR acquisitions with a small time lag and manual image registration[3].

In this paper, we present a new technique for comparing sea ice classification from different SAR acquisitions respecting the underlying drift. Moreover, the evaluated drift field is used to identify potential areas of sea ice deformation, which in turn can influence the quality of the sea ice classification. For this reason we combine our software processors for sea ice drift and classification.

\section{METHODOLOGY}

The comparison of two or more SAR scenes, enables the estimation of the sea ice drift with high resolution. The resulting drift field is used to relate different ice areas within two temporal separated SAR scenes. Independent from the drift, sea ice can be grouped into classes depending on the age and appearance of the ice. Using the drift field, changes of sea ice areas and limitations of the existing classification can be analysed. Figure 1 shows our process chain for creating a superposition of two sea ice classification results based on independent SAR products. In the first step, sea ice drift and classification are performed independently. After this, the classification results from the initial scene are superimposed on the subsequent scene using a co-registration and drift compensation step.

In the following, the dataset used and the steps of our processing chain are described in detail.

\subsection{Dataset}

Our tests deals with ten TerraSAR-X (TS-X) Stripmap Dualpol MGD scenes acquired over the Arctic Ocean north of 
Greenland. From these ten acquisitions we matched ten pairs of co-located scenes with a maximum acquisition time difference of about 26 hours. Table 1 lists the TS-X products used. In the following text the image pairs are denoted due to the combination of the single scenes indices (e.g. 01|02).

\subsection{Drift estimation}

The sea ice drift is estimated from pairs of co-located SAR scenes. Firstly, both scenes are co-registrated based on geospatial information. For each designated drift vector $\vec{v}_{k}$ a patch $g_{x_{k} y_{k}}$ from the initial scene and a patch $h_{x_{k}+u_{k} y_{k}+v_{k}}$ from the subsequent scene is extracted. Each patch has a fixed pixel size $N$ and is referenced by its upper left corner coordinate $(x, y)$ of the corresponding scene. The parameters $u_{k}, v_{k}$ describing the vertical and horizontal components of the drift vector initialized with $(0,0)$. On the extracted patches, a phase correlation method is applied to estimate the drift vector. The normalized cross spectrum $\Gamma(i, j)$ is estimated from the Fourier-transformed image patches $\mathcal{F}(g)$ and $\mathcal{F}(h)$ :

$$
\Gamma(i, j)=\mathcal{F}^{-1}\left(\frac{\mathcal{F}(g) \mathcal{F}(h)}{|\mathcal{F}(g) \mathcal{F}(h)|}\right)
$$

The peak in the cross spectrum indicates the drift vector. Next, the position of the image patch $h$ is updated to the estimated drift vector and the phase correlation method is repeated. Reiterating this procedure until the peak is estimated in the centre of the cross spectrum, the optimum drift vector is found. In Addition to this iterative method, a multiresolution image pyramid is applied. The update of all drift vectors in four resolution levels with a reduction factor of two and starting with the coarsest resolution enables sea ice drift estimation from large displacement to small changes in the ice field. A more detailed description of the drift estimation is given in [1].

\subsubsection{Deformation}

Changes in drift velocities can result in deformed ice areas. In general, deformed sea ice is related to a rougher surface, which in turn effects in a stronger SAR backscatter. For this reason, deformed sea ice can often not be distinguished from older sea ice which in general shows a higher backscatter. The common way to extract deformation zones is to calculate the partial derivative $\partial u / \partial x$ and $\partial v / \partial y$ from the estimated drift field and determine in a second step the amount of convergence and divergence within the drift field [4].

$$
\nabla \vec{v}(x, y)=\frac{\partial u}{\partial x}+\frac{\partial v}{\partial y}
$$

This makes it possible to distinguish between sea ice areas of homogeneous drift and zones of potential ice deformation.

\subsection{Sea ice classification}

In [2], we presented a supervised sea ice classification algorithm based on SAR Polarimetry and an Artificial Neuronal Network (ANN). From complex co-polarization channels $S_{H H}$ and $S_{V V}$ of TS-X Dualpol Stripmap products, we create the covariance matrix $C_{c o}$.

$$
C_{c o}=\left[\begin{array}{cc}
S_{H H}^{2} & S_{H H} S_{V V} \\
S_{H H} S_{V V} & S_{V V}^{2}
\end{array}\right]
$$

Based on the covariance matrix, we calculated polarimetric features as an input for the ANN. For training of the ANN, we chose rectangular patches from selected scenes and validated our results with in situ data. Due to the absence of complex TS-X products for near real-time (NRT) usage, we adjusted our algorithm for the non-complex Dualpol Stripmap MGD product. Therefore the number of features is reduced using only features depending on the amplitude of the polarization channels. After this, the ANN was re-trained based on the patches from the MGD products and the in situ data mentioned in [2]. Similar to our previous work, the adjusted algorithm differentiates four ice classes, open water (OW), young ice (YI), smooth first year ice (SFYI), and rough firstyear and multiyear ice (RFYMYI). The denotation indicates that first year ice is, depending on its appearance, classified into SFYI or RFYMYI. Due to changes in the surface roughness and the influence of the incidence angle, a differentiation between first year ice and multiyear ice is often challenging.

\subsection{Superposition of sea ice classification}

For creating a pixelwise superposition of sea ice classification results, each pixel needs to be assigned drift information.

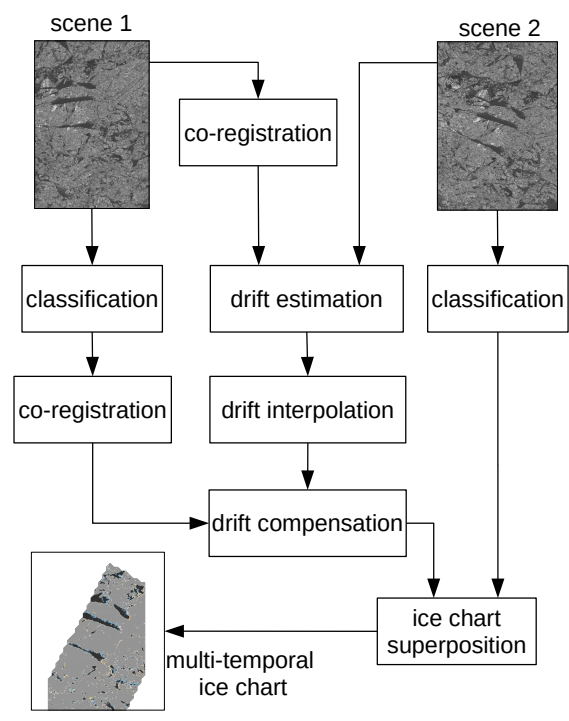

Fig. 1: Flowchart of superposition creation 
Table 1: List of TS-X-Dual-Polarimetric StripMap Scenes

\begin{tabular}{cccccc}
\hline Index & Time UTC & Beam mode & Incid. ang. & Center loc. & Orbit \\
\hline 01 & March 05, 2018, 16:19:42 & stripFar_009 & $35.5^{\circ}-36.6^{\circ}$ & $85.5 \mathrm{~N},-48.1 \mathrm{E}$ & Ascending \\
02 & March 06, 2018, 16:02:31 & stripNear_009 & $34.2^{\circ}-35.6^{\circ}$ & $85.4 \mathrm{~N},-47.0 \mathrm{E}$ & Ascending \\
03 & March 15, 2018, 16:36:53 & stripNear_009 & $34.2^{\circ}-35.6^{\circ}$ & $85.2 \mathrm{~N},-49.2 \mathrm{E}$ & Ascending \\
04 & March 15, 2018, 18:11:08 & stripFar_013 & $42.7^{\circ}-43.8^{\circ}$ & $84.8 \mathrm{~N},-45.0 \mathrm{E}$ & Ascending \\
05 & March 15, 2018, 18:11:16 & stripFar_013 & $42.7^{\circ}-43.8^{\circ}$ & $85.5 \mathrm{~N},-48.8 \mathrm{E}$ & Ascending \\
06 & March 16, 2018, 16:19:44 & stripFar_008 & $31.1^{\circ}-34.5^{\circ}$ & $85.2 \mathrm{~N},-49.3 \mathrm{E}$ & Ascending \\
07 & March 16, 2018, 17:53:58 & stripFar_012 & $41.0^{\circ}-42.2^{\circ}$ & $84.9 \mathrm{~N},-43.1 \mathrm{E}$ & Ascending \\
08 & March 16, 2018, 17:54:04 & stripFar_012 & $41.0^{\circ}-42.2^{\circ}$ & $84.2 \mathrm{~N},-47.7 \mathrm{E}$ & Ascending \\
09 & September 05, 2018, 06:08:11 & stripFar_026 & $58.9^{\circ}-59.5^{\circ}$ & $89.9 \mathrm{~N}, 44.5 \mathrm{E}$ & Descending \\
10 & September 05, 2018,09:17:40 & stripNear_025 & $57.4^{\circ}-58.1^{\circ}$ & $88.9 \mathrm{~N}, 44.0 \mathrm{E}$ & Descending \\
\hline
\end{tabular}

For the reason that pixelwise drift estimation would cause a long processing time we interpolate the estimated drift field to pixel resolution in two steps. Firstly, from the estimated drift vectors a triangular grid is created using a Delaunay triangulation. After this, each pixel within the triangular grid is interpolated due to the three drift vectors at the vertices of the corresponding grid cell. Using this approach, the interpolated drift field can be created independent from the alignment and structure of the previous estimated drift field. Moreover, insufficient drift vectors can easily be skipped from the drift interpolation, resulting in a coarser grid resolution. Finally the sea ice classification from initial image is shifted by the interpolated drift field.

\section{RESULTS AND DISCUSSION}

In general, the changes identified in our classification results can be caused by three principal mechanisms. Firstly, uncertainties in the drift estimation can cause false change detections close to the edges between different types of ice. Secondly, the appearance of an ice type has really changed due to meteorological effects like wind, deformation, thaw or freeze. Lastly, changes in classification are caused by limitations of the classification algorithm itself.

In the following, the superposition of the classification results are analysed in two respects. Firstly, the general reliability of the classification results is evaluated for homogeneous areas of ice movement (section 3.1). Secondly, converging and diverging zones of the drift field are analysed regarding changes in classification (section 3.2).

\subsection{Analysis of classification reliability}

In the selected region and season, the generated sea ice classification shows for all ten scenes a dominant presence of RFYMYI. Figure 2 shows different processing steps for image pair $05 \mid 08$. Figure 2(a) shows the co-registered scene of the initial acquired product, 2(b) shows the subsequent scene with the drift field overlaid, 2(c) illustrates the estimated zones of converging and diverging sea ice, and 2(d) points out the estimated sea ice classes in grayscale and the indicated changes overlaid in colour. Noticeable in Figure 2(d) are the uniformly distributed changes of small sea ice areas between SFYI and RFYMYI. The changes are marked in yellow and blue. Physically, a change from first year ice to multiyear ice within the maximum time difference of 26 hours is not possible. Moreover, changes due to deformation or uncertainties of the drift algorithm can be neglected because of the homogeneous drift. Therefore, we assume that the vast majority of these changes result from limitations in the classification algorithm. Due to similar roughness and the influence of the incidence angle, SFYI and RFYMYI can often not be distinguished. The analysis of the other image pairs confirms the existence of these noise-like ice class changes caused by limitations of the classification algorithm. Applying image filter algorithms as a post processing step of the classification would help to overcome these limitations. However, using filter algorithms would also erase other small class areas which could indicate existing deformed ice such as pressure ridges or small open water areas. Therefore creating the superposition of two or more sea ice classification images can be used to detect and overcome these limitations without losing information from the high resolution.

\subsection{Influence of deformation}

Convergent ice movement can cause deformation and result in a rougher surface, which in turn can change of the appearance of the ice in SAR and results in misclassification of our algorithm. Therefore areas of converging sea ice are analysed in more detail. In the centre of Figure 2(b) the local velocity of the sea ice is decreasing in the flow direction, which creates converging zones. To analyse the reliability of our classification in the potential deformation zones, we estimate the amount of changes separately for homogeneous and converging drift areas. The image pairs $05|08,05| 06,04 \mid 07$ and 


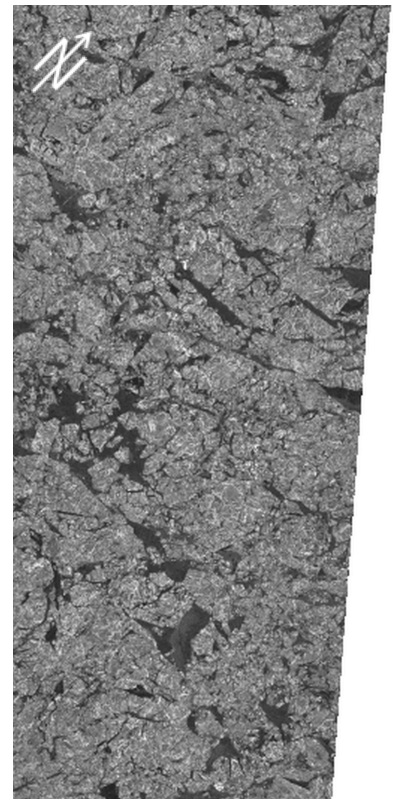

(a)

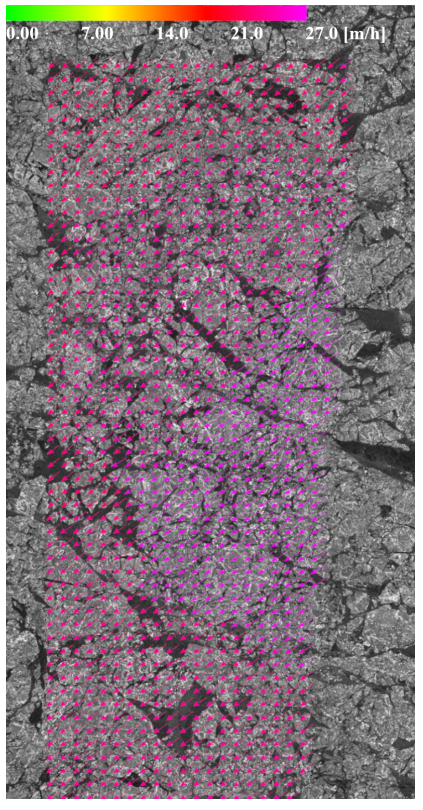

(b)

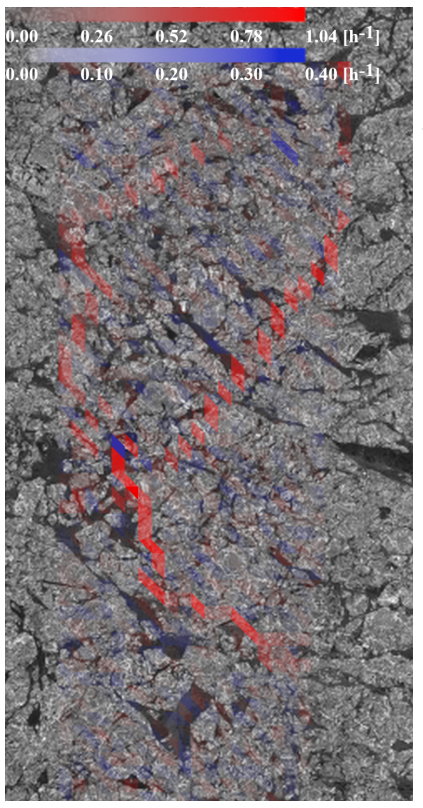

(c)

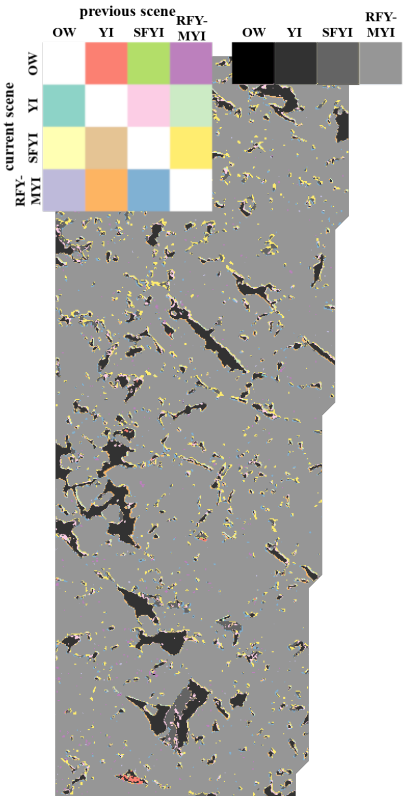

(d)

Fig. 2: (a) Scene 05 co-registered to 08 (b) Scene 05 with estimated sea ice drift field (c) Highlighted convergence and divergence zones in $h^{-1}$ (convergence in red, divergence in blue), divergence (d) Grayscaled sea ice classification from scene 08 and colour-coded class changes compared to classification based on scene 05

$03 \mid 06$ have a common drift velocity from $22 \mathrm{~m} / \mathrm{h}$ to $27 \mathrm{~m} / \mathrm{h}$. Therefore, these pairs are suitable for comparing the distribution of ice class changes within and outside of converging ice zones. Based on the convergence map of the image pairs, the ice is separated into areas of homogeneous movement and potential deformation areas. A convergence of $0.4 h^{-1}$ is used as a threshold for convergence areas. In general, the amount of ice class changes within the converging zones are higher than in areas of homogeneous motion. In Figure 2(c) the estimated convergence and divergence areas of the pair $05 \mid 08$ are shown. Within the four mentioned image pairs the amount of sea ice changes from YI to RFYMYI is increasing in average by $6.2 \%$. Analysing all sea ice types, the amount of changes is $11 \%$ higher. This confirms our expectations.

\section{CONCLUSION}

In this paper we presented a new approach for a pixelwise superposition of two sea ice classification results from different independent SAR acquisitions. This offers the opportunity to analyse changes within the ice and the reliability of our classification algorithm. Moreover, we superimposed drift information to identify potential areas of ice deformation and demonstrated the influence to our algorithm. In future we plan to extend the afore mentioned technique for a series of SAR products and use the estimated information for bordering different reasons of ice type changes. Finally, this might increase the reliability of our operational sea ice processors.

\section{REFERENCES}

[1] Anja Frost, Stefan Wiehle, Suman Singha, and Detmar Krause, "Sea ice motion tracking from near real time sardata acquired during antarctic circumnavigation expedition,” in IGARSS 2018, IEEE, Ed. Juli 2018, IEEE, pp. $1-4$, IEEE Xplore.

[2] Rudolf Ressel, Suman Singha, Susanne Lehner, Anja Rosel, and Gunnar Spreen, "Investigation into different polarimetric features for sea ice classification using x-band synthetic aperture radar," IEEE Journal of Selected Topics in Applied Earth Observations and Remote Sensing, vol. 9, no. 7, pp. 3131-3143, jul 2016.

[3] A. Malin Johansson, Camilla Brekke, Gunnar Spreen, and Jennifer A. King, "X-, c-, and l-band SAR signatures of newly formed sea ice in arctic leads during winter and spring," Remote Sensing of Environment, vol. 204, pp. 162-180, jan 2018.

[4] Gunnar Spreen, Ron Kwok, Dimitris Menemenlis, and An T. Nguyen, "Sea-ice deformation in a coupled ocean-sea-ice model and in satellite remote sensing data," The Cryosphere, vol. 11, no. 4, pp. 1553-1573, jul 2017. 